\title{
Laminin and Optic Nerve Regeneration in the Goldfish ${ }^{1}$
}

\author{
J. M. HOPKINS $\ddagger$, T. S. FORD-HOLEVINSKI $\ddagger$, J. P. McCOY $\S$, AND B. W. AGRANOFF* $\ddagger^{2}$ \\ Departments of *Biological Chemistry, $\Varangle$ Psychiatry, and $\$$ Pathology, University of Michigan, Ann Arbor, Michigan 48109
}

\begin{abstract}
Previous work from our laboratory had shown that goldfish retinal fragments explanted onto a polylysine substratum 1 to 2 weeks following optic nerve crush exhibit a striking clockwise pattern of neuritic outgrowth. In the present study, however, when the basal lamina component laminin was used as a substratum, neurites grew out as uncurved spokes, were less fasciculated, and had an increased rate of elongation. When laminin was combined with polylysine in the substratum, the degree of fasciculation and rate of elongation were similar to those seen on laminin alone, whereas the tendency for clockwise outgrowth was even more pronounced than that observed with polylysine alone. These results suggest that regenerating neurites have an affinity for laminin. Using an antibody to murine Engelbreth-HolmSwarm sarcoma laminin, which cross-reacted with basal lamina in goldfish tissue sections, we studied the histochemical distribution of laminin in the goldfish visual system. Immunoperoxidase staining for laminin showed a characteristic scalloped pattern of staining in cross-sections of optic nerve bundles. Following optic nerve crush, the reaction product became much more diffuse and intense, especially distal to the crush site. When the retinal ganglion cell bodies were eliminated by removing the eye, the degenerating optic nerve stump still showed the intensive staining. We interpret these results to indicate that optic nerve glia are responsible in large part for the formation of laminin. Taken together, these in vivo and in vitro findings suggest that laminin plays a role in nerve regeneration in the goldfish central nervous system.
\end{abstract}

The basis for the profound difference in the ability of nerves to regenerate in the peripheral nervous system (PNS) compared with those in the central nervous system (CNS) is of great theoretical as well as practical significance. Axotomy in the adult avian or mammalian CNS is followed by abortive sprouting of the affected nerves and formation of a glial scar, whereas injured peripheral nerves

Received February 19, 1985; Revised April 8, 1985;

Accepted April 10, 1985

${ }^{1}$ We thank Ms. M. Lewis for performing the nerve crushes, Mr. W. Schade and G. Merz for technical assistance in laminin staining. Ms. S. Dunham for preparing the manuscript, and Drs. S. Easter and P. Raymond for critically reviewing it. This research was sponsored by National Institutes of Health Grant NS 13743 and National Eye Institute Grant EY 05947. T. S. F.-H. was supported by National Institutes of Health Training Grant MH 15794, and J. $\mathrm{M}$. H. is a predoctoral trainee, supported by National Institutes of Health Grant 5 T32 MH 14279 in Neuroscience. A preliminary report of this work was presented at the 1984 meeting of the Society for Neuroscience

${ }^{2}$ To whom correspondence should be addressed, al Neuruscience Lab Building, University of Michigan, 1103 East Huron, Ann Arbor, Ml 48109. commonly regenerate and make functional reconnections (Kiernan, 1978; Aguayo et al., 1982). Experiments in which injured axons from CNS have been shown to regenerate over long distances through PNS autografts (Richardson et al., 1980; Aguayo et al., 1982; Richardson and Issa, 1984) suggest that CNS neurons have the intrinsic ability to regenerate, if they are given a permissive environment. Attention has thus been directed at the extraneuronal environment, including supporting cells and the extracellular matrix (ECM), as potential determinants of regenerative capacity (Collins, 1978; Letourneau, 1979; Carbonetto et al., 1983; Edgar et al., 1984). ECM components which have been implicated include collagen, laminin (LAM), and fibronectin.

LAM, a major component of the basement membrane, has been characterized as a 900-kilodalton cruciform glycoprotein and is known to mediate cell-cell and cell-substratum interactions (Timpl et al., 1979). It has also been shown to support neurite extension from a variety of neuronal cell types in culture (Rogers et al., 1983; Liesi et al., 1984; Smalheiser et al., 1984). In some mammalian tissues, LAM has been localized by high resolution immunohistochemistry to the lamina lucida (lamina rarae) of the basal lamina (Gospodarowicz et al,, 1981; Sanes, 1982; Martinez-Hernandez and Chung, 1984). In Schwann tubes of the mammalian PNS, the basal lamina is asymmetric, with the collagen-rich lamina densa facing outward and the LAM-rich lamina lucida facing inward (Carbonetto, 1984).

In morphological studies, Scherer and Easter (1984) showed that the growth cones of the goldfish trochlear nerve are almost always found in close association with the inside surface of Schwann tubes. In the adult goldfish retina, new ganglion cells are constantly added at the retinal margin, and emerging growth cones maintain continuous contact with basal lamina starting from the inner limiting membrane, then along the retinal artery, and finaliy along the pial surface of the optic nerve (Easter et al., 1981, 1984).

These findings suggest that ECM-associated LAM may facilitate regeneration in the goldfish CNS and PNS by providing an adhesive surface to support and guide the advancing growth cone. Since the goldfish visual system differs from that of birds and mammals in that it regenerates following optic nerve axotomy, it was of interest to us to see whether it also differed in its LAM content and whether axotomy altered its distribution.

\section{Materials and Methods}

Animals. Goldfish (Carassius auratus) 6 to $7 \mathrm{~cm}$ in body length (Ozark Fisheries, Stoutland, MO) were maintained at $25^{\circ} \mathrm{C}$ and fed daily. Intraorbital crush of the right optic nerve was performed as previously described (Springer and Agranoff, 1977). Malc Spraguc-Dawley rats (180 to $190 \mathrm{gm}$ ) were obtained from Spartan Research Animals, Inc. (Haslett, Ml) and given food and water ad libitum.

Preparation of tissue cullure substrala and cullure media. Plastic 35-rnm culture dishes (Nunclon, Vanguard International, Neptune, $N J$ ) were treated with polylysine (PLYS) and/or LAM to produce three substrata: PLYS, PLYS + LAM, and LAM. LAM was either the gift of Mr. R. Knibbs and Dr. I. Goldstein, University of Michigan, or was purchased from Bethesda Research 

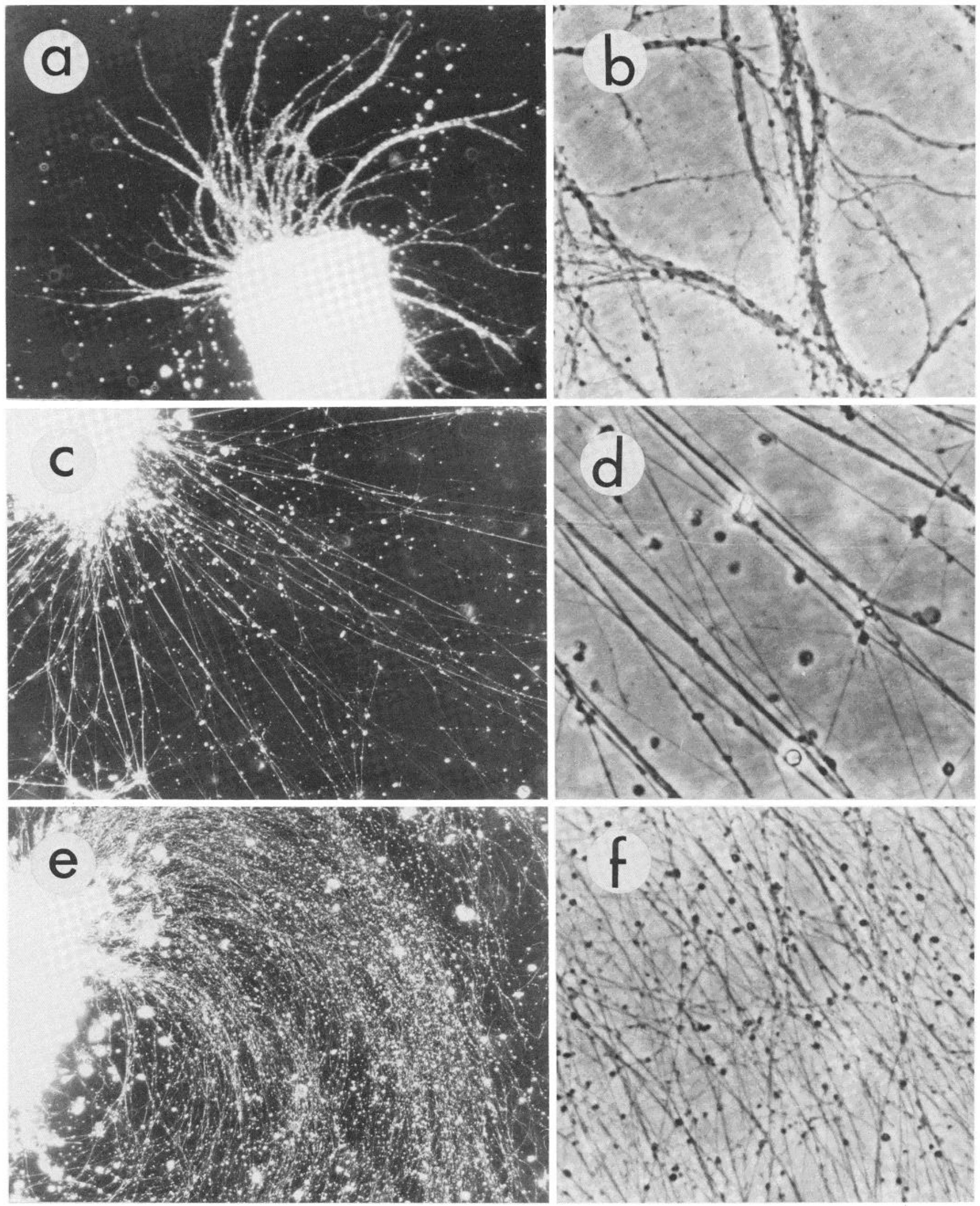

Figure 1. Morphology of neurite outgrowth and fasciculation in vitro. a, c, and e depict explants from PC goldfish retina as seen with pseudo-darkfield illumination. Magnification $\times 40$. The bright square is the explant body, from which neurites can be seen growing out onto the substratum. $b, d$, and $f$ are phase contrast views of the neurites. Magnification $\times 430$. The explant shown in a and $b$ was grown on PLYS, that in $c$ and $d$ was grown on purified LAM, and that in $e$ and $f$ was grown on PLYS + LAM. 

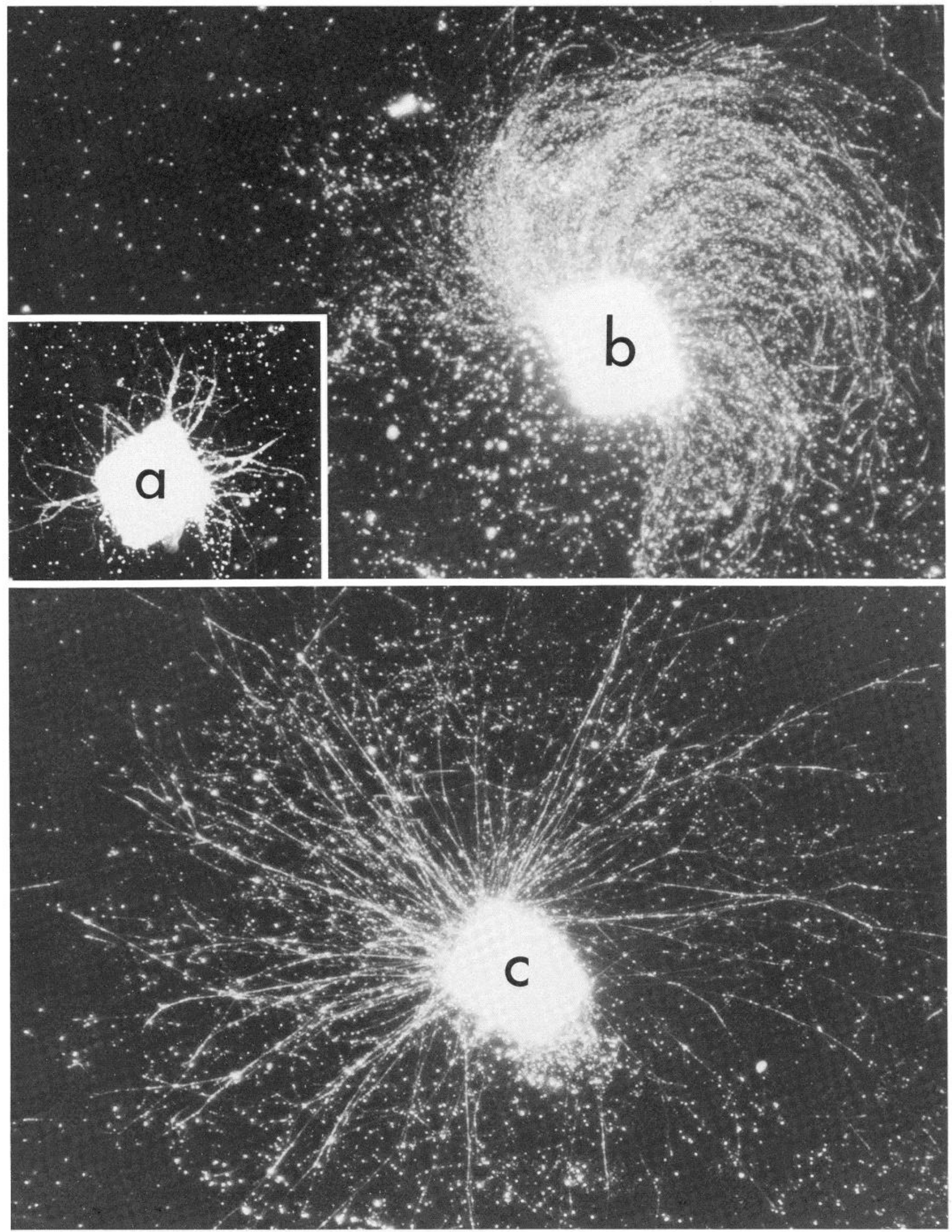

Figure 2. Comparison of neurite outgrowth and morphology from explants of goldfish retinas. a, PLYS substratum ( 8 days PC, 6 days in vitro); $b$, PLYS + LAM substratum ( 16 days PC, 3 days in vitro); $c$, LAM substratum ( 16 days PC, 3 days in vitro). Magnification $\times 40$. 
Laboratories (Bethesda, MD). PLYS (type IV Sigma Chemical Co., St Louis, $\mathrm{MO}$ ) was applied to sterile dishes by incubating them overnight at $20^{\circ} \mathrm{C}$ with $1 \mathrm{mg} / \mathrm{ml}$ of PLYS in sodium borate buffer, $0.2 \mathrm{M}, \mathrm{pH}$ 8.4. LAM substrata were prepared by incubating dishes with a solution of LAM (100 or $40 \mu \mathrm{g} /$ $\mathrm{ml})$ in the borate buffer overnight at $20^{\circ} \mathrm{C}$. PLYS + LAM substrates were prepared by incubating PLYS-coated dishes with a LAM solution $(10 \mu \mathrm{g} / \mathrm{ml}$ unless otherwise noted). All dishes were rinsed extensively with sterile distilled water after the incubation. Leibowitz L-15 nutrient medium was supplemented with $20 \mathrm{~mm}$ Na HEPES (hydroxyethylene piperazine ethanesulfonate, Sigma) buffer, $\mathrm{pH} 7.4,0.1 \mathrm{mg} / \mathrm{ml}$ of gentamicin sulfate (Sigma), $0.1 \mathrm{~mm} 5^{\prime}$-fluorodeoxyuridine (Sigma), $0.2 \mathrm{~mm}$ uridine (Sigma), and $1 \%$ fetal calf serum (FCS: Grand Island Biological Co., Grand Island, NY). In previous studies, we employed the same mixture, with the exception that $10 \%$ FCS was used (Heacock and Agranoff, 1977).

Culture of retinal explants. Retinal explants, $500 \mu \mathrm{m}$ on a side, were prepared with a Mcllwain tissue chopper as previously described (Landreth and Agranoff, 1976). Pooled explants prepared from six to eight retinas of fish in which the optic nerves had been crushed 7 to 14 days before explanation (post-crush, PC) were used for each experiment, unless otherwise noted.

Estimation of neurite outgrowth. The extent and morphology of neuritic outgrowth from each explant was determined under pseudo-darkfield or phase contrast optical conditions using a Leitz Diavert microscope. Each cxplant was scorcd 0 to +4 for density of ncuritic outgrowth by comparison with a photographic atlas (Landreth and Agranoff, 1979); neuritic length was measured with an ocular micrometer by positioning the explant under the relicle scale. The numerical producl of densily and length yielded a nerve growth index (Landreth and Agranoff, 1979). To measure the length of highly curved neurites such as were seen with some PLYS cultures, and especially with PLYS + LAM cultures, the field was positioned sequentially under the reticle using arbitrary landmarks on the neurite.

Tissue sectioning. Goldfish were anesthetized with tricaine methanesulfonate and decapitated; then, the skull cap was removed. The brain, optic nerves, and eyes were removed together and frozen in $M-1$ embedding medium (Lipshaw, Detroit, Ml) on dry ice. In some instances, the optic nerves (from the optic disk up to, and including, the tectum) were dissected out of unoperated and PC fish and placed side by side on a block of frozen embedding medium. These were then covered with additional embedding medium and frozen on dry ice. Rats were asphyxiated in a carbon dioxide environment and decapitated. The brains were removed and embedded as above. Blocks were trimmed and sliced into 8- $\mu \mathrm{m}$ sections which were placed on gelatin-subbed slices, air-dried, and stored at $4^{\circ} \mathrm{C}$.

Antibody preparation. Antisera directed against LAM (puritied from Engelbreth-Holm-Swarm (EHS) murine sarcoma) were prepared by the repeated injection of the antigen into New Zealand White rabbits. Rabbit antiserum to bovine serum albumin (BSA) was the gift of Dr. K. J. Johnson, University of Michigan. Affinity-purified antibodies were prepared from each antiserum by column chromatography, using an appropriate Sepharose-antigen matrix. The affinity-purified antibodies were examined for purity by SDS-gel electrophoresis and by immunoelectrophoresis. Antibody specificity was assessed by enzyme-linked immunosorbent assay (McCoy et al., 1983), immunodiffusion (Ouchteriony and Nilsson, 1978), and immunoperoxidase reaction (described below) on appropriate tissue sections.

Immunocytochemistry. Immunoperoxidase staining was accomplished using an avidin-biotin complex (ABC) method (Hsu et al., 1981). Mounted sections of either rat or goldfish tissue were fixed in cold acetone $\left(4^{\circ} \mathrm{C}\right)$ for $10 \mathrm{~min}$. The sections were then air-dried for $5 \mathrm{~min}$, followed by rehydration in phosphate-buffered saline (PBS), preincubated with normal horse serum (1:20 in PBS) for $10 \mathrm{~min}$ at room temperature, and then incubated for $30 \mathrm{~min}$ with appropriately diluted crude or affinity-purified antibodies. Slides were washed three times with PBS and then were incubated with biotinylated antirabbit immunoglobulin (Vectastain Kit, Vector Laboratories, Burlingame, CA). Following the $30-\mathrm{min}$ incubation at $25^{\circ} \mathrm{C}$, the slides were washed three times in PBS and treated with 1.5\% hydrogen peroxide in $50 \%$ aqueous methanol to remove endogenous peroxidase activity. The slides were again washed three times in PBS and were then incubated for $30 \mathrm{~min}$ at room temperature with $\mathrm{ABC}$ reagent (Vectastain Kit, Vector Laboratories). The slides were subsequently washed three times in PBS and then were incubated with a solution of $0.1 \%$ diaminobenzidine and $0.015 \% \mathrm{H}_{2} \mathrm{O}_{2}$ in $\mathrm{PBS}$ to visualize the distribution of the complex. The slides were washed twice in distilled water, counterstained with hematoxylin, rinsed in tap water, dehydrated, and mounled wilh coverslips.

\section{Results}

In vitro studies. Squares of goldfish retina, explanted onto PLYS coated dishes 1 to 2 weeks after a "conditioning" lesion to the optic nerve, extend neurites after 1 or 2 days in culture which characteristically grow out in a clockwise pattern of large fascicles (Heacock and Agranoff, 1977; Fig. 1, $a$ and $b$ ). When such retinal explants were cultured on LAM $(100 \mu \mathrm{g} / \mathrm{ml})$, the rate of neurite elongation was dramatically increased over that seen in PLYS controls (Figs. 2 and 3). The pattern of outgrowth on LAM was radial and nearly devoid of curvature (Fig. 1, $c$ and $d$ ), whereas the estimated degree of fasciculation and density of outgrowth were reduced compared with those seen in PLYS dishes. To conserve the supply of LAM, we next incubated dishes previously coated with PLYS overnight with a $10-\mu \mathrm{g} / \mathrm{ml}$ solution of LAM. Unexpectedly, neurite outgrowth on the combined PLYS + LAM substratum showed an accentuation of the clockwise curvature characteristic of growth on PLYS alone (Fig. 1e). However, the neurites exhibited the reduced degree of fasciculation that was seen on pure LAM (Fig. 1f). Due to the differences in apparent fasciculation, the densities of outgrowth on PLYS and on PLYS + LAM are difficult to compare, although growth in both of these conditions appeared to be more dense than growth seen on LAM alone (Fig. 2). After taking into account differences in curvature, the rate of neurite elongation on PLYS + LAM was similar to that seen on LAM (Fig. 3). To determine the optimal concentration
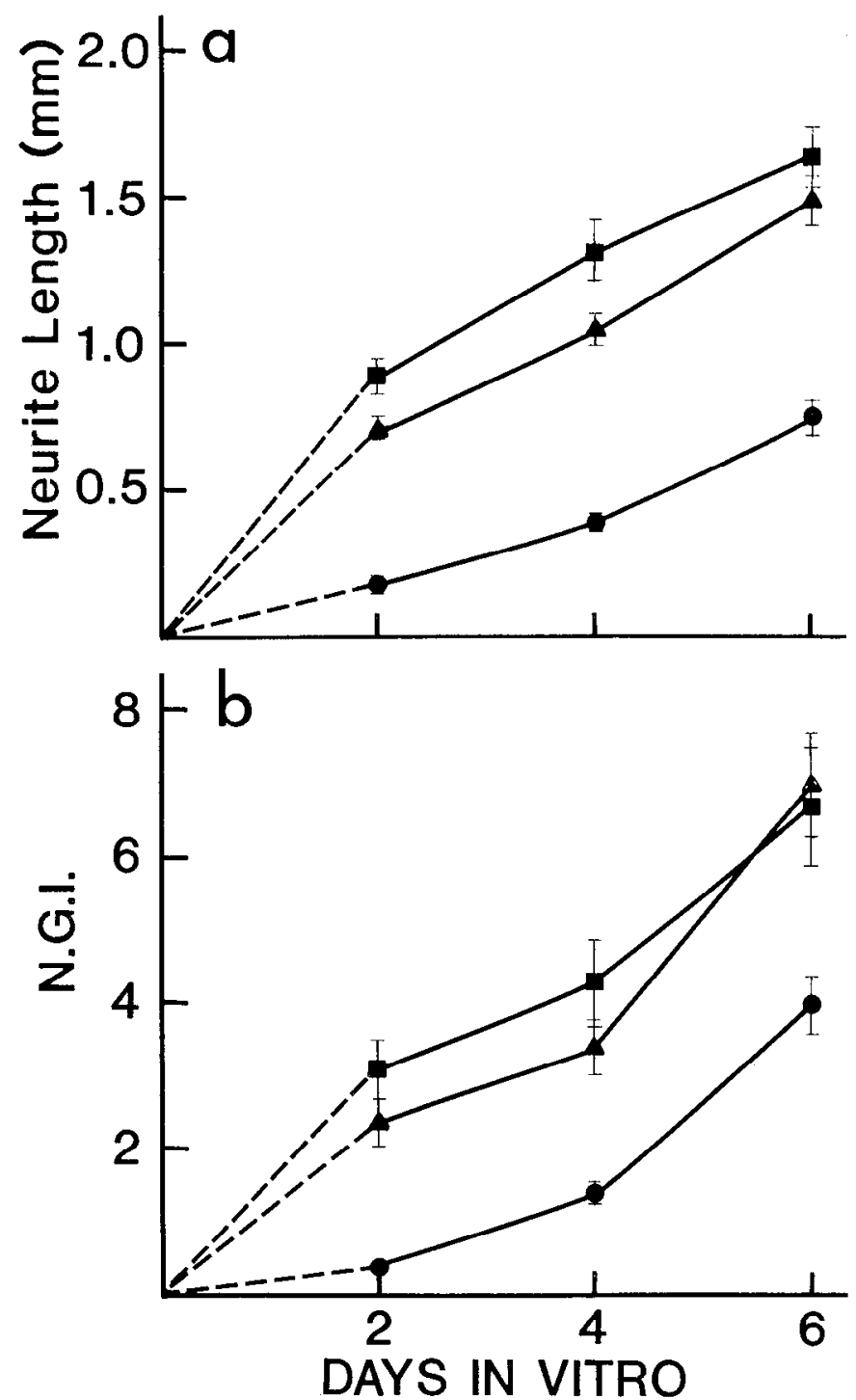

Figure 3. Effect of substrate composition on neurite elongation and nerve growth index in vitro. a, Neurite length in millimeters as a function of time in culture. $b$, Nerve growth index as a function of time in culture. Each data point represents an average, calculated from explants showing growth \pm SEM. - PLYS; $\boldsymbol{A}$. PLYS + LAM; $\mathbf{\square}$, LAM. (See also Table I.) 
of LAM for the preparation of our PLYS + LAM substrate, a series of PLYS-coated dishes was incubated overnight at $20^{\circ} \mathrm{C}$ with 1,2 , 5 , or $10 \mu \mathrm{g} / \mathrm{ml}$ of LAM. The rate of neurite elongation from PC explants, when compared after 1 day in culture, was concentration dependent between 1 and $5 \mu \mathrm{g} / \mathrm{ml}$ and reached a plateau at $10 \mu \mathrm{g} /$ $\mathrm{ml}$ of LAM (data not shown). The latter concentration of LAM was therefore used for coating dishes in further studies.

Explant attachment and number of attached explants showing neurite outgrowth were somewhat lower in LAM cultures, compared with PLYS or PLYS + LAM cultures (Table I). Measurements of growth indicated that the effect of LAM on neurite elongation was greatest in the first few days of culture. At early time points, neurites grown in the presence of LAM grew 5 to 8 times more rapidly than those grown on PLYS alone (Fig. 3). Neurite outgrowth in LAM and PLYS + LAM cultures was detected within $6 \mathrm{hr}$ of explantation, the earliest time examined, whereas in PLYS cultures, emerging neurites are not generally seen until 24 to $48 \mathrm{hr}$ post-explantation.

Explants from retinas which had not received a conditioning lesion typically exhibited scanty neurite outgrowth which appeared after 7 days in culture on a PLYS substratum. The presence of LAM in the substratum did not stimulate the appearance of neurite outgrowth from these explants.

In the present and previous experiments, we have tested the neurite-promoting effects of various substratum-bound materials, including components of the ECM. Collagen, a prominent component of the ECM, is known to support neurite outgrowth in this system (Landreth and Agranoff, 1976), although it is not as effective as PLYS. In the present studies fibronectin, an ECM protein functionally related to LAM (Mosher and Furcht, 1981), had no apparent effect on neurite outgrowth when used at $20 \mu \mathrm{g} / \mathrm{ml}$ in combination with PLYS (data not shown). It should be noted here that fetal calf serum typically contains about $20 \mu \mathrm{g}$ of fibronectin per milliliter (M. Wicha, personal communication). All of our cultures were therefore grown in the presence of about $0.2 \mu \mathrm{g} / \mathrm{ml}$ of fibronectin. Neither BSA nor a high molecular weight fraction of goldfish brain supported explant attachment. LAM is thus the only ECM component we have tested which has greater neurite-promoting abilities than the synthetic (PLYS) substratum.

In vivo studies. The dramatic increase observed in the neurite elongation rate in the presence of LAM prompted us to examine normal and regenerating optic nerves for the occurrence of this protein. An antibody raised against LAM purified from mouse EHS sarcoma (Timpl et al., 1979) gave an immunoperoxidase staining pattern characteristic of LAM distribution in peripheral nerve (Bignami et al., 1984a) when used to stain goldfish trigeminal nerve sections (Fig. 4). When cross-sections of goldfish optic nerves were examined at 28 days $\mathrm{PC}$, the injured nerve exhibited strikingly more immunoreactive material than did the control (Fig. 5a). A previous morpho-

TABLE I

Effect of time in culture and substratum composition on attachment of explants and neurite outgrowth

The percentage of the total number of explants cultured that were attached to the substratum $(\% \mathrm{~A})$ and the percentage of attached explants showing neurite outgrowth $(\% \mathrm{G})$ are given. Data are from the experiment shown in Figure 3. Forty-five explants were cultured in the PLYS + LAM group, 45 in the LAM group, and 27 in the PLYS group.

\begin{tabular}{cccc}
\hline & \multicolumn{3}{c}{ Days In Vitro } \\
\cline { 2 - 4 } & 2 & 4 & 6 \\
\hline PLYS & 93 & 93 & 93 \\
$\%$ A & 72 & 92 & 78 \\
$\%$ G & & & \\
PLYS + LAM & 96 & 89 & 84 \\
$\%$ A & 70 & 87 & 82 \\
$\%$ G & & & \\
LAM & 69 & 76 & 78 \\
$\%$ A & 61 & 68 & 70 \\
$\%$ G & & & \\
\hline
\end{tabular}
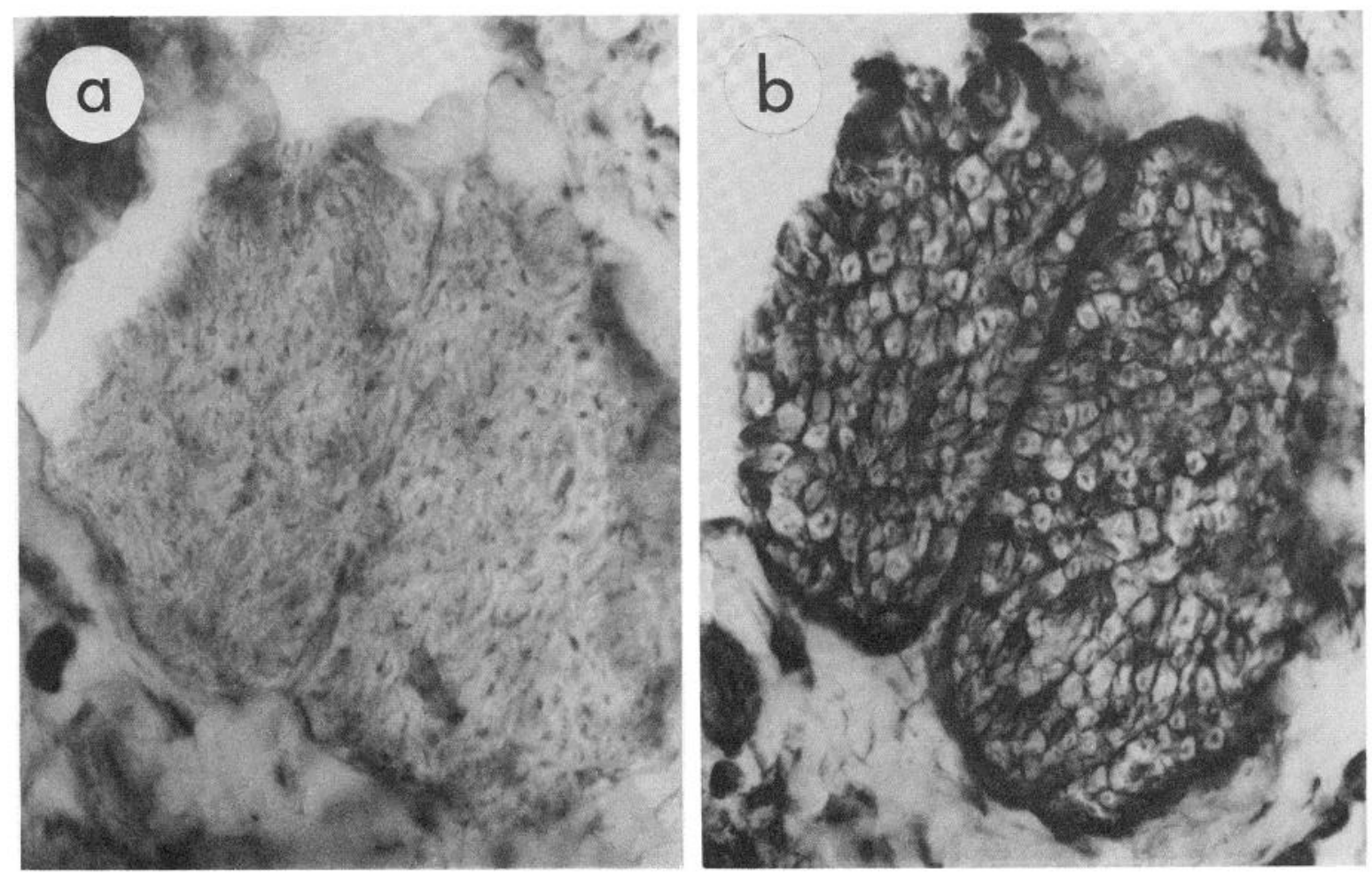

Figure 4. Immunoperoxidase-stained frozen sections of adult goldfish trigeminal nerve after binding of rabbit preimmune whole serum (a) or whole serum from rabbits immunized against LAM isolated from murine EHS sarcoma (b). The nerve was folded prior to embedding to increase cross-sectional area. Note the ringlet pattern characteristic of peripheral nerve endoneurial tubes in the anti-LAM-stained section. Magnification $\times 400$. 
logical observation that the basal lamina of the goldfish invaginates, dividing the nerve into large bundles (Lanners and Grafstein, 1.980; Wolburg, 1981), was confirmed immunohistochemically. It was also apparent in the 28-day PC nerve that staining within the large nerve bundles was more diffuse and greatly increased when compared with control nerves (Fig. 5, $b$ and $c$ ). In the unlesioned rat optic nerve, LAM appeared to be confined to the epineurial sheath and vascular endothelium (Fig. $5 d$ ).

Since it appeared that LAM-reactive material was present in the goldfish optic nerve, and that its concentration increased after the nerve was crushed, we wanted to know whether the time course of this increase corresponded to degenerative changes or to fiber regeneration and functional recovery. Accordingly, optic pathways from unoperated fish and from fish in which the right optic nerve had been lesioned were sectioned and immunostained at various times PC. In these experiments, the removed nerves were uncrossed at the optic chiasm and sectioned longitudinally. Affinity-purified antibody was used to reduce nonspecific background staining. Nerves from unoperated goldfish exhibited very low background staining with this antibody but still showed discrete regions of intense reactivity over blood vessels or basal lamina (Fig. 5e). At 37 days PC the lesioned optic nerve reacted intensely to the antibody on both sides of the crush site (Fig. $5 f$ ), whereas at 11 days PC (Fig. $5 \mathrm{~g}$ ) the increased staining was seen only distal to the crush site. The density of staining was similar to that of controls at 3 days PC, whereas the increase appeared to be diminishing at 52 days PC (not shown). To learn more about the cellular source of the increased LAM staining following crush, the right eye was removed in a group of fish, thus eliminating any ganglion cell contribution to the LAM in the optic nerve. When the degenerating stump was examined 7 days later (Fig. $5 h$ ), the increase in LAM-reactive material observed was similar to that scen when the nerve was crushed and the eye was not removed.

In each experiment, adjacent sections were reacted with affinitypurified anti-BSA. This antibody was prepared in the same fashion as the anti-LAM and was used as a control for specification of staining and of binding. In each instance, little or no peroxidase reaction product was observed.

\section{Discussion}

Recent interest in the nature of the ECM, particularly in developing and regenerating nerves (Carbonetto et al., 1983; Lander et al., 1983; Rogers et al., 1983; Carbonetto, 1984) raised the question of its possible role in the regenerating goldfish optic nerve. Of particular interest is LAM, a basement membrane component, which has been characterized biochemically (Timpl et al., 1979). The present finding that LAM indeed serves as an excellent substratum in that it enhances the rate of neurite outgrowth from retinal explants suggests that the neurites themselves may bind specifically to this molecule. Although it is perhaps overly simplistic to equate rate of neurite outgrowth in vitro with efficacy of the regeneration process, it is impressive that the measured rate of elongation on LAM seen in Figure 3 was 5 times that seen on PLYS. Furthermore, the rate of outgrowth observed on LAM was approximately equivalent to that seen for regeneration in vivo at $20^{\circ} \mathrm{C}$, i.e., about $6 \mathrm{~mm}$ in 20 to 30 days (Springer and Agranoff, 1977; Davis et al., 1984). None of the other substrata (or combinations) tested thus far support as high a rate of outgrowth.

The observed effect of LAM on fasciculation suggests that the affinity of neurites for LAM exceeds that between neurites. Noble et al. (1984) have shown that the fasciculation of neurites growing out from dissociated fetal rat cerebellar and spinal neurons in vitro is dependent on the type of non-neuronal cell substrate on which they are grown. The degree of fasciculation observed is high on a lawn of fibroblasts, whereas neurites grown on astrocytes or Schwann cells show none. LAM is produced by Schwann cells (Cornbrooks et al., 1983) and astrocytes (Liesi et al., 1983) in primary culture but is not made by fibroblasts. Thus, the presence or absence of LAM could explain these effects on fasciculation.
The fact that the initiation of neurite emergence from the explant was accelerated in the presence of LAM may be an indication of enhanced attachment of filopodia to the substratum. Embryonic chick ciliary ganglion neurons in culture extend filopodia which do not immediately adhere to a PLYS substrate. Within minutes after the addition of heart-conditioned medium, they can be seen to adhere firmly, followed immediately by neurite extension (Collins, 1978). It is possible that the observed neurite-promoting effects of many previously described conditioned media, including heart-conditioned media, are in fact mediated by LAM or LAM-proteoglycan complexes (Lander et al., 1983, 1984). Although LAM was clearly effective in stimulating neurite elongation in the present study, it appeared less effective than PLYS in effecting explant attachment, perhaps due to differences in nonspecific adhesive interactions.

Growing neurites may have a higher affinity for LAM than for PLYS, and this neurite-LAM adhesivity may be mediated by cell surface receptors. In other systems, such as human MCF-7 breast carcinoma cells and mouse fibrosarcoma cells, such associations are believed to be mediated by a specific membrane protein (Malinoff and Wicha, 1983; Terranova et al., 1983). LAM receptors may also participate in cytoskeletal organization and intracelluar transport by binding actin filaments within the cell by a transmembrane mechanism (Brown et al., 1983). Another possible receptor for laminin is heparan sulfate proteoglycan (HSP), a molecule with a known binding site on LAM (Sakashita et al., 1980) and which has already been shown to be rapidly transported in the goldfish optic nerve (Elam and Agranoff, 1971). Edgar et al. (1984) have shown that the heparin-binding domain of the LAM molecule is both necessary and sufficient for the outgrowth of neurites from chick sympathetic neurons. Since HSP is also thought to possess a transmembrane segment with a binding site for actin and is thought to bind LAM at the heparin-binding site (Rapraeger and Bernfield, 1982), it is a promising candidate for a role in the interaction of cytoskeletal elements with their external environment. Involvement of glycosylated proteins in neurite growth is further supported by the finding that tunicamycin, an inhibitor of protein glycosylation, inhibits neurite outgrowth from goldfish retinal explants (Heacock, 1982).

The hypothesis drawn from these experiments, that LAM plays a role in regeneration, is reinforced by our observations on LAM distribution in normal and regenerating optic nerve in vivo. The fortunate finding that goldfish basement membranes cross-reacted with antibody to mouse tumor LAM permitted us to examine the presence of LAM immunohistochemically. The distribution pattern of LAM-immunoreactive material in the goldfish optic nerve reflects the known location of basal lamina seen by transmission electron microscopy (Lanners and Grafstein, 1980; Wolburg, 1981). Unlike mammalian optic nerve (Fig. 5d), connective tissue containing fibrocytes, collagenous fibers, and blood vessels divides the goldfish optic nerve into large bundles. These are further subdivided by astrocytes which also produce the basal lamina that separates the nerve fibers in the large bundles from the septa of connective tissue (Wolburg, 1981). During optic nerve growth, which occurs throughout adulthood as ganglion cells are added at the periphery of the retina, new growth cones push aside the glial endfeet and older axons to remain adjacent to this basal lamina from the retina to the optic chiasm (Easter et al., 1984). Thus, in normal optic nerve the growing neurites appear to be associated specifically with the LAMcontaining basal lamina.

During regeneration a similar association may occur. Following nerve crush, the lissue is invaded by macrophages and microglia. There is also a proliferation of astrocytes and, to a lesser degree, of oligodendrocytes. The astrocytes extend processes deep into the nerve, and when new bundles of unmyelinated axons grow through the nerve they abut these processes and occasionally those of the oligodendrocytes (Wolburg, 1981). Since the astrocytes already possess the capacity to produce basal lamina, it is possible that they are altered during degeneration and express unstructured LAM on their surfaces, thereby increasing the area of adhesive contact available to the invading growth cones. This may account for the 


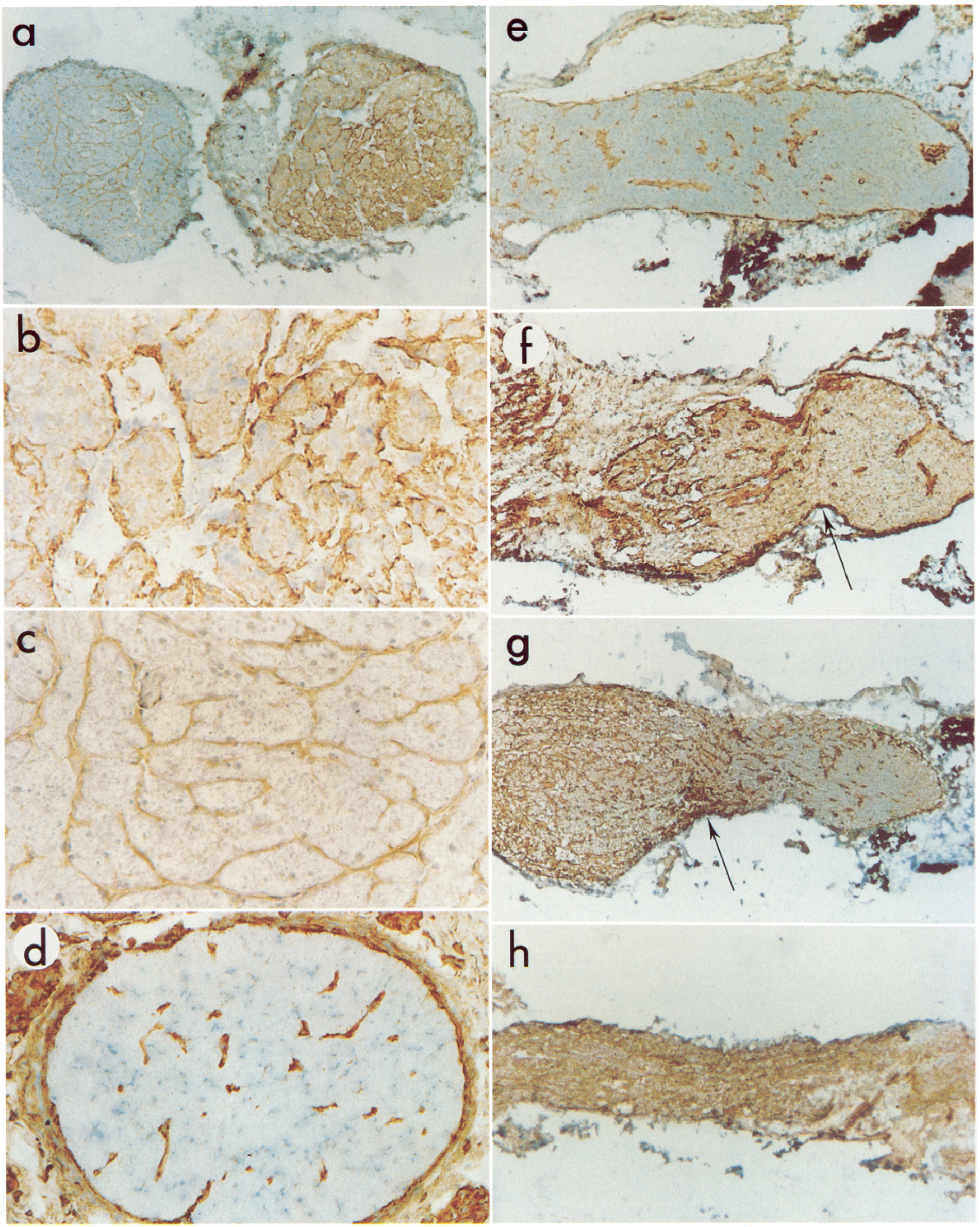


apparent homogeneous increase in background staining of damaged and regenerating nerves seen by immunohistochemistry at the light microscopic level (Fig. 5b). This increase in LAM background staining could also reflect its release and diffusion from degenerating basement membrane. This seems unlikely, since basement membrane appears to remain intact in studies on degenerating peripheral nerve (Bignami et al., 1984a). A preliminary ultrastructural study of degenerating goldfish optic nerve suggests that the basal lamina surrounding the large bundles also remains intact, although its profile in cross-section becomes convoluted due to shrinkage of fiber bundles ( $\mathrm{S}$. Easter, personal communication).

The time course of the observed increase in LAM immunoreactivity in the optic nerve parallels that observed for invasion of the nerve by regenerating axons. However, the increase first appears distal to the site of crush, remains prominent long after morphological and functional recovery, and, in the case of the enucleation experiment, occurs even in the absence of axon regrowth. For these reasons we conclude that new axons are not a significant source of the LAM increase nor do they act as a signal for its production. It is more likely that the glia are stimulated to produce LAM either by the disappearance of an axonally transported inhibitor, by the appearance of a degeneration-associated substance, or both. In support of the second mechanism, Giulian (1984) has reported that a soluble peptide fraction isolated from denervated goldfish tecta stimulates incorporation of labeled thymidine and amino acids into isolated optic tract glia.

The persistence of LAM after a conditioning lesion is not surprising in light of the 2 to 3 months required for the architecture of the nerve to return to normal (Wolburg, 1981). The state of the degenerating nerve with respect to glial proliferation and a prolonged increased in LAM availability could, in part, explain the observed acceleration of functional recovery following a second lesion of the same nerve (McQuarrie and Grafstein, 1981; R. E. Davis, personal communication).

It remains possible that macrophages contribute to the observed increase in LAM immunoreactivity. This cell type remains in the nerve for approximately 2 months following crush (Wolburg, 1981) and, although it is thought to possess a receptor for LAM, its ability to express this molecule is still in doubt (Wicha and Huard, 1983). Resolution of this question may require experiments at the electron microscopic level.

The ability to producc LAM in response to injury has bcen considered to be a hallmark of the PNS. This distinction from the CNS is evident in a recent study by Bignami et al. (1984b) in which injury-induced stimulation of LAM immunoreactivity was found to be confined to the peripheral course of the regenerating dorsal root in the rat spinal cord. The goldfish optic nerve is then an example of CNS tissue which behaves like PNS tissue, in that it produces LAM in response to injury. We conclude that this injury-induced increase in LAM may facilitate the rapid regrowth of retinal ganglion cell axons through the goldfish optic nerve, and suggest that this, in conjunction with the possible intrinsic properties that permit the retinal ganglion cells to regrow their axons (Benowitz et al., 1981; Skene and Willard, 1981; Agranoff and Ford-Holevinski, 1984), is responsible for the high regenerative capacity of this tissue. The relationship between intrinsic properties of neurons and the extraneuronal milieu, and their relative contributions to functional restoration remain important experimental questions that have significant clinical implications.

\section{References}

Agranoff, B. W., and T. S. Ford-Holevinski (1984) Biochemical aspects of the regenerating goldfish visual system. In Advances in Neurochemistry. Vol. 6: Axonal Transport in Neuronal Growth and Regeneration, J. S. Elam and P. Cancalon, eds., pp. 69-86, Plenum Press, New York.

Aguayo, A., S. David, P. Richardson, and G. Bray (1982) Axonal elongation in peripheral and central nervous system transplants. Adv. Cell. Neurobiol. 3: 215-234.

Benowitz, L. I., V. E. Shashoua, and M. G. Yoon (1981) Specific changes in rapidly transported proteins during regeneration of the goldfish optic nerve. J. Neurosci. 1: 300-307

Bignami, A., N. H. Chi, and D. Dahl (1984a) Laminin in rat sciatic nerve undergoing Wallerian degeneration. An immunofluorescent study with neurofilament and laminin antisera. J. Neuropathol. Exp. Neurol. 43: 94103.

Bignami, A., N. H. Chi, and D. Dahl (1984b) Regenerating dorsal roots and the nerve entry zone: An immunofluorescent study with neurofilament and laminin antisera. Exp. Neurol. 85: 426-436.

Brown, S. S., H. L. Malinoff, and M. S. Wicha (1983) Connectin: Cell surface protein that binds both laminin and actin. Proc. Natl. Acad. Sci. U. S. A. 80: $5927-5930$.

Carbonetto, S. (1984) The extracellular matrix of the nervous system. Trends Neurosci. 7: 382-387.

Carbonetto, S., M. M. Gruver, and D. C. Turner (1983) Nerve fiber growth in culture on fibronectin, collagen, and glycosaminoglycan substrates. J. Neurosci. 3: 2324-2335

Collins, F. (1978) Axon initiation by ciliary neurons in culture. Dev. Biol. 65. 50-57.

Cornbrooks, C. J., D. J. Carey, J. A. McDonald, R. Timpl, and R. P. Bunge (1983) In vivo and in vitro observations on laminin production by Schwann cells. Proc. Natl. Acad. Sci. U. S. A. 80: 3850-3854.

Davis, R. E., B. E. Schlumpf, and P. D. Klinger (1984) Systemic colchicine inhibits goldfish optic nerve regeneration. Toxicol. Appl. Pharmacol. 73: 268-274.

Easter, S. S., Jr., A. C. Rusoff, and P. E. Kish (1981) The growth and organization of the optic nerve and tract in juvenile and adult goldfish. $J$. Neurosci. 1: 793-811

Easter, S. S., Jr., B. Brallon, and S. S. Scherer (1984) Growth-related order of the retinal fiber layer in goldfish. J. Neurosci. 4: 2173-2190.

Edgar, D., R. Timpl, and H. Thoenen (1984) The heparin-binding domain of laminin is responsible for its effects on neurite outgrowth and neuronal survival. EMBO J. 3: 1463-1468.

Elam, J. S., and B. W. Agranoff (1971) Transport of proteins and sulfated mucopolysaccharides in the goldfish visual system. J. Neurobiol. 2: 379390.

Gospodarowic7, D., G. Greenburg, J. M. Foidart, and N. Savion (1981) The production and localization of laminin in cultured vascular and cornea endothelial cells. J. Cell. Physiol. 107: 171-183.

Giulian, D. (1984) Peptides from the regenerating central nervous system of goldfish stimulate glia. Proc. Natt. Acad. Sci. U. S. A. 81: 3567-3571.

Heacock, A. M. (1982) Glycoprotein requirement for neurite outgrowth in goldfish retinal explants: Effects of tunicamycin. Brain Res. 241: 307-315.

Heacock, A. M., and B. W. Agranoff (1977) Clockwise growth of neurites from retinal explants. Science 198: 64-66

Hsu, S. M., L. Raine, and H. Fanger (1981) The use of avidin-biotin-peroxidase complex in immunoperoxidase techniques: A comparison between $\mathrm{ABC}$ and unlabeled antibody (PAP) procedures. J. Histochem. Cytochem. 29: $577-580$

Kiernan, J. A. (1978) An explanation of axonal regeneration in peripheral nerves and its failure in the central nervous system. Med. Hypotheses 4 $15-26$

Lander, A. D., K. Tomaselli, A. L. Calof, and L. F. Reichardt (1983) Studies on extracellular matrix components that promote neurite outgrowth. Cold Spring Harbor Symp. Quant. Biol. 48: 611-623.

Figure 5. Immunohistochemical localization of anti-LAM antibody in frozen sections of optic nerve. LAM-reactive material is brown. Sections are counterstained with hematoxylin. $a$, Cross-section of goldfish optic nerves removed 28 days after crush of the right nerve $(P C)$. This section was made distal to the site of crush and was stained using whole anti-LAM serum. Magnificatıon $\times 60$. $b$, Magnification of the PC nerve shown in a. Note the peroxidase staining within the large bundles, as well as accentuated staining around bundles. Magnification $\times 300$. $c$, The control nerve exhibits peroxidase staining primarily in the region surrounding the bundles. Magnification $\times 300 . d$. Cross-section of frozen rat optic nerve reacted with whole anti-LAM serum. Note peroxidase staining within the nerve is confined to blood vessels. Magnification $\times 120$. e, Optic nerve from an unoperated goldfish. All longitudinal sections are shown with the optic disk to the right and are reacted with affinity-purified anti-LAM. $f$, goldfish optic nerve at 37 days PC. The site of crush is indicated by the arrow. $e$ and $f$ are taken from the same slide and are shown at the same magnification $(\times 75)$. g. Goldfish optic nerve 11 days PC. The arrow indicates the site of crush. Note that at 37 days PC the LAM staining is uniform, whereas at 11 days PC it is greatest distal to the site of crush (to the left of the arrow). Magnification $\times 50 . h$, Goldfish optic nerve 7 days following enucleation. Magnification $\times 50$. 
Lander, A. D., D. K. Fujii, D. Gospodarowicz, and L. F. Reichardt (1984) "Neurite outgrowth-promoting factors" in conditioned media are complexes containing laminin. Soc. Neurosci. Abstr. 10:40

Landreth, G. E., and B. W. Agranoff (1976) Explant culture of adult goldfish retina: Effect of prior optic nerve crush. Brain Res. 118: 299-303

Landreth, G. E., and B. W. Agranoff (1979) Explant culture of adult goldfish retina: A model for the study of CNS regeneration. Brain Res. 161:39-53.

Lanners, H. N., and B. Grafstein (1980) Effect of a conditioning lesion on regeneration of goldfish optic axons: Ultrastructural evidence of enhanced outgrowth and pinocytosis. Brain Res. 196: 547-553.

Letourneau, P. C. (1979) Cell-substratum adhesion of neurite growth cones, and its role in neurite elongation. Exp. Cell Res. 124: 127-138.

Liesi, P., D. Dahl, and A. Vaheri (1983) Laminin is produced by early rat astrocytes in primary culture. J. Cell Biol. 96: 920-924.

Liesi, P., D. Dahl, and A. Vaheri (1984) Neurons cultured from developing rat brain attach and spread preferentially to tarninin. J. Neurosci. Res. 11: 241-251.

Malinoff, H. L., and M. S. Wicha (1983) Isolation of a cell surface receptor protein for laminin trom murine tibrosarcoma cells. J. Cell Biol. 96: 14751479.

Martinez-Hernandez, A., and A. E. Chung (1984) The ultrastructural localization of two basement membrane components: Entactin and laminin in rat tissues. J. Histochem. Cytochem. 32: 289-298.

McCoy, J. P., D. Schrier, E. J. Lovett, W. J. Judd, and J. Varani (1983) Hyperimmune human $\mathrm{ABO}$ blood typing sera: Reactivity with murine laminin and cytotoxicity for metastatic murine tumor cells. J. Cell Sci. 59: 245-256.

McQuarrie, I. G., and B. Grafstein (1981) Effect of a conditioning lesion on optic nerve regeneration in goldfish. Brain Res. 216: 253-264.

Mosher, D. F., and L. T. Furcht (1981) Fibronectin: Review of its slructure and possible functions. J. Invest. Dermatol. 77: 175-180.

Noble, M., J. Fok-Seang, and J. Cohen (1984) Glia are a unique substrate for the in vitro growth of central nervous system neurons. J. Neurosci. 4 : $1892-1903$

Ouchterlony, O., and L. A. Nilsson (1978) Immunodiffusion and immunoelectrophoresis. In Handbook of Experimental Immunology, D. M. Weir, ed., Vol. 1, pp. 19.1-19.44, Blackwell Scientific Publishers, Oxford.
Rapraeger, A. C., and M. Bernfield (1982) An integral membrane proteoglycan is capable of binding components of the cytoskeleton and the extracellular matrix. In Extracellular Matrix, pp. 265-269, Academic Press, Inc., New York.

Richardson, P. M., and V. M. K. Issa (1984) Transplantation of embryonic spinal and cerebral tissue to sciatic nerves of adult rats. Brain Res. 298: $146-148$

Richardson, P. M., U. M. McGuiness, and A. J. Aguayo (1980) Axons from CNS neurones regenerate into PNS grafts. Nature 284: 264-265.

Rogers, S. L., P. C. Letourneau, S. L. Palm, J. McCarthy, and L. T. Furcht (1983) Neurite extension by peripheral and central nervous system neurons in response to substratum-bound fibronectin and laminin. Dev. Biol. 98: $212-220$

Sakashita, S., E. Engvall, and E. Ruoslahti (1980) Basement membrane glycoprotein laminin binds to heparin. FEBS Lett. 116: 243-246.

Sanes, J. R. (1982) Laminin, fibronectin, and collagen in synaptic and extrasynaptic portions of muscle fiber basement membrane. J. Cell Biol. 93: $442-451$.

Scherer, S. S., and S. S. Easter, Jr. (1984) Degenerative and regenerative changes in the trochlear nerve of goldfish. J. Neurocytol. 13: 519-565.

Skene, J. H. P., and M. Willard (1981) Changes in axonally transported proteins in toad retinal ganglion cells. J. Cell Biol. 89: 86-95.

Smalheiser, N. R., S. M. Crain, and L. M. Reid (1984) Laminin as a substrate for retinal axons in vitro. Dev. Brain Res. 12: 136-140.

Springer, A. D., and B. W. Agranoff (1977) Effect of temperature on rate of goldfish optic nerve regeneration: A radioautographic and behavioral study. Brain Res. 128: 405-415.

Terranova, V. P., C. N. Rao, T. Kalebic, I. M. Margulies, and L. A. Liotta (1983) Laminin receptor on human breast carcinoma cells. Proc. Natl. Aciad. Sci. U. S. A. 80: 444-448.

Timpl, R., H. Rohde, P. G. Robey, S. I. Rennard, J. -M. Foidart, and G. R. Martin (1979) Laminin-A glycoprotein from basement membranes. J Biol. Chem. 254: 9933-9937.

Wicha, M. S., and T. K. Huard (1983) Macrophages express cell-surface laminin. Exp. Cell Res. 143: 475-479.

Wolburg, H. (1981) Axonal Transport, Degeneration, and Regeneration in the Visual System of the Goldfish. Adv. Anat. Embryol. Cell Biol. 67: 1-94. 